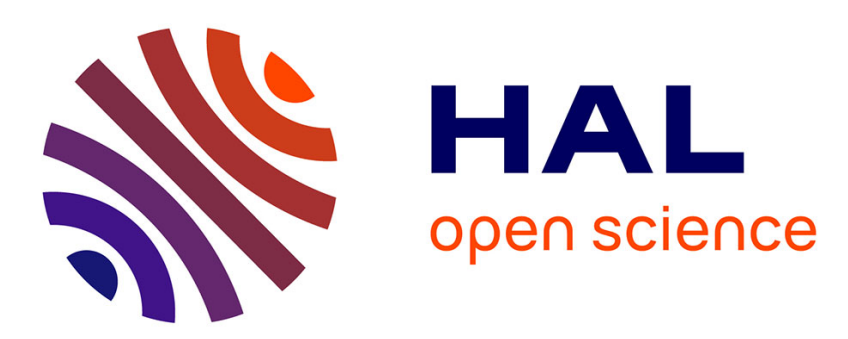

\title{
A High-Performance Frequency Stability Compact CPT Clock Based on a Cs-Ne Microcell
}

R. Boudot, X. Liu, P. Abbe, R.K. Chutani, N. Passilly, S. Galliou, C. Gorecki, V. Giordano

\section{- To cite this version:}

R. Boudot, X. Liu, P. Abbe, R.K. Chutani, N. Passilly, et al.. A High-Performance Frequency Stability Compact CPT Clock Based on a Cs-Ne Microcell. IEEE Transactions on Ultrasonics, Ferroelectrics and Frequency Control, 2012, 59 (11), pp.2584-2587. 10.1109/TUFFC.2012.2493 . hal-00776544

\section{HAL Id: hal-00776544 https://hal.science/hal-00776544}

Submitted on 18 Apr 2021

HAL is a multi-disciplinary open access archive for the deposit and dissemination of scientific research documents, whether they are published or not. The documents may come from teaching and research institutions in France or abroad, or from public or private research centers.
L'archive ouverte pluridisciplinaire HAL, est destinée au dépôt et à la diffusion de documents scientifiques de niveau recherche, publiés ou non, émanant des établissements d'enseignement et de recherche français ou étrangers, des laboratoires publics ou privés.

\section{(c)(1)}

Distributed under a Creative Commons Attribution| 4.0 International License 


\section{A High-Performance Frequency Stability Compact CPT Clock Based on a Cs-Ne Microcell}

\author{
Rodolphe Boudot, Xiaochi Liu, Philippe Abbé, \\ Ravinder Chutani, Nicolas Passilly, Serge Galliou, \\ Christophe Gorecki, and Vincent Giordano
}

\begin{abstract}
This paper reports on a compact table-top Cs clock based on coherent population trapping (CPT) with advanced frequency stability performance. The heart of the clock is a single buffer gas Cs-Ne microfabricated cell. Using a distributed feedback (DFB) laser resonant with the Cs $D_{1}$ line, the contrast of the CPT signal is found to be maximized around $80^{\circ} \mathrm{C}$, a value for which the temperature dependence of the Cs clock frequency is canceled. Advanced techniques are implemented to actively stabilize the clock operation on a zero-light-shift point. The clock frequency stability is measured to be $3.8 \times 10^{-11}$ at $1 \mathrm{~s}$ and well below $10^{-11}$ until 50000 s. These results demonstrate the possibility to develop highperformance chip-scale atomic clocks using vapor cells containing a single buffer gas.
\end{abstract}

\section{INTRODUCTION}

$\mathrm{T}$ HE combination of coherent population trapping (CPT) physics [1] and micro electromechanical systems (MEMS) allows the development of chip-scale atomic clocks (CSAC) [2] exhibiting a volume of a few cubic centimeters, a power consumption of about $100 \mathrm{~mW}$, and a fractional frequency stability better than $10^{-11}$ at 1 day or even 1 week integration time [3]. Such frequency references, outperforming crystal quartz oscillators over long time scales, are intended to provide excellent base timing for numerous battery-operated portable applications, such as telecommunication networks synchronization, navigation, military systems, or even power distribution and underwater sensing [4].

The heart of a miniature atomic clock consists of a hermetically sealed microfabricated cell containing alkali vapor and a buffer gas atmosphere. On the one hand, the buffer gas is required to reduce the $\mathrm{CPT}$ resonance broadening resulting from depolarizing wall collisions and to eliminate Doppler broadening through Lamb-Dicke

R. Boudot, X. Li, P. Abbé, S. J. Galliou, and V. Giordano are with Franche-Comté Electronique Mécanique Thermique Optique-Sciences et Technologies (FEMTO-ST), Time and Frequency Department, Centre National de la Recherche Scientifique (CNRS), Besançon, France (e-mail: rodolphe.boudot@femto-st.fr).

R. Chutani, N. Passilly, and C. Gorecki are with Franche-Comte Electronique Mécanique Thermique Optique-Sciences et Technologies (FEMTO-ST), Micro-Nano Sciences and Systems (MN2S) Department, Centre National de la Recherche Scientifique (CNRS), Besançon, France. narrowing [5]. On the other hand, the buffer gas causes a temperature-dependent buffer gas pressure collisional frequency shift of the clock transition. Traditionally, the temperature coefficient of the frequency shift is reduced by mixing two buffer gases, each of which shifts the clock frequency in opposite ways [6]-[8]. This technique is widely used in commercially available optically pumped devices. However, such a mixture is difficult to control accurately in MEMS cells because of their small size and the high temperatures involved in the anodic bonding process required for their fabrication.

Most microfabricated cells are basically realized by etching of a silicon wafer and subsequent anodic bonding of a thin glass wafer to each side [9]. Our filling method differs from traditional ones [10], [11] in that the Cs vapor is generated only after complete sealing of the cell by local heating of a side-cavity Cs metallic dispenser with a highpower infrared laser source [12]. This method is intended to reduce interactions with highly-reactive Cs chemistry and to achieve a strong bonding quality [13]. Moreover, we recently reported the first direct observation of a quadratic dependence on temperature of the Cs clock transition around a so-called inversion temperature $T_{\mathrm{inv}}$ of about $80^{\circ} \mathrm{C}$ in presence of a single Ne buffer gas [14]. The inversion temperature, found to be similar in a pulsed Cs CPT clock using centimeter-scale cells [15], was measured to be independent of the Ne buffer gas pressure. These results emerged as significantly interesting for the development of simple-configuration high-performance miniature clocks and justified deep characterizations of CPT resonances in Cs-Ne microcells [16].

The short-term frequency stability of a passive atomic clock is improved by both reducing the detected resonance linewidth and increasing its signal-to-noise ratio. The best reported short-term frequency stabilities of millimeterscale Cs-cell-based CPT clocks are measured to be 1 to 3 $\times 10^{-10}$ at $1 \mathrm{~s}$ when operated with a laser resonant on the $\mathrm{D}_{2}$ line [2] and are improved to 2 to $4 \times 10^{-11}$ [3], [11] by using the $\mathrm{D}_{1}$ line. For integration times typically higher than $1000 \mathrm{~s}$, the clock frequency is subject to drift. This is mainly due to collisional frequency shifts and light shift phenomena. The first issue is solved by stabilizing the cell temperature close to the inversion temperature, whereas the total light shift can be canceled by fine adjustment of the RF modulation index [17]-[20]. An additional servo loop can then be implemented to lock the clock to the point of minimum light shift [21]-[23].

In this paper, we present a compact CPT clock prototype based on a Cs microfabricated cell filled with $\mathrm{Ne}$, combined with an externally-modulated DFB laser resonant with the $\mathrm{Cs}_{1} \mathrm{D}_{1}$ line. Implementation of advanced techniques leads to frequency stability performance comparable to those of the best microcell-based CPT clocks. These results prove for the first time that our Cs-Ne cell 


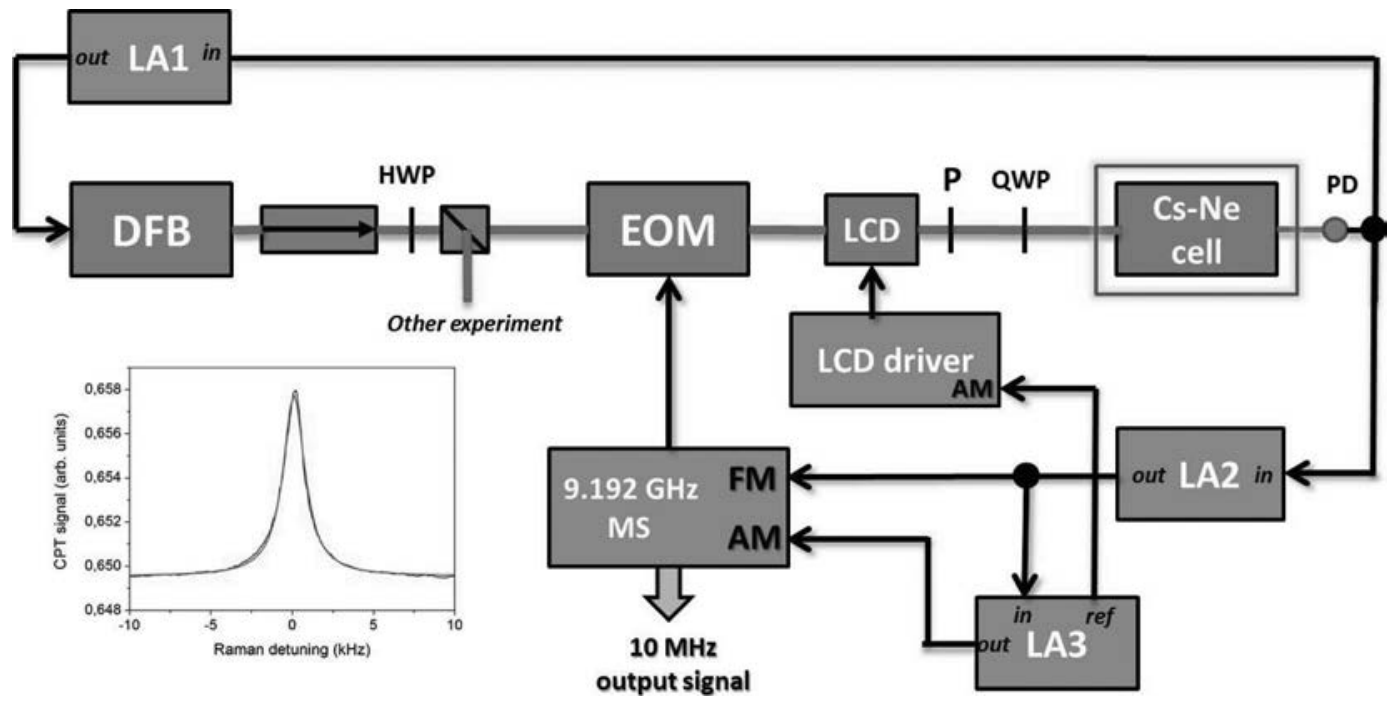

Fig. 1. Schematic of the Cs-Ne microcell-based coherent population trapping (CPT) clock. MS: 10-MHz quartz-oscillator-based microwave synthesizer; EOM: electro-optic modulator; LCD: voltage-controlled liquid crystal plate attenuator; P: polarizer; HWP: half-wave plate; QWP: quarter-wave plate; LA1: lock-in-amplifier-based servo for distributed feedback (DFB) laser frequency stabilization through modulation at $60 \mathrm{kHz}$ of the laser bias current; LA2: lock-in-amplifier-based servo for locking the local oscillator frequency to the CPT resonance transition, LA3: lock-in-amplifier-based servo for light shift correction (LSC).

technology solution is compatible with the development of miniature atomic clocks exhibiting high long-term frequency stability performance.

\section{Experimental Setup}

Fig. 1 presents the Cs CPT clock experimental setup. The laser source is a commercially-available GaAs semiconductor DFB laser diode emitting at $894.6 \mathrm{~nm}$ on the Cs $\mathrm{D}_{1}$ line. A Faraday optical isolator is placed at the output of the DFB laser to avoid optical feedback. Optical sidebands required to perform CPT spectroscopy are generated by driving an external pigtailed phase electrooptic modulator (EOM) at $9.192 \mathrm{GHz}$ using a microwave frequency synthesizer inspired from [24]. The fiber modulator ensemble is inserted into a box with insulating foam and is actively temperature-stabilized to the millikelvin level to reduce output laser intensity fluctuations. No phase noise degradation was observed between the microwave frequency synthesizer output and the 9.192-GHz optically carried signal. The 2-mm-diameter collimated laser beam is circularly polarized using a quarter-wave plate. The laser power is $17 \mu \mathrm{W}$ before entering the atomic resonator. The atomic resonator consists of a $2-\mathrm{mm}-$ diameter and 1.4-mm-long cylindrical microfabricated Cs vapor cell filled with Ne. The cell temperature $T_{\text {cell }}$ is stabilized close to $80^{\circ} \mathrm{C}$, which corresponds to a Cs density of $4.05 \times 10^{12}$ atoms $/ \mathrm{cm}^{3}$. Temperature fluctuations of the microcell are below the millikelvin level. A static magnetic field of a few microteslas parallel to the laser beam propagation direction is applied to split the hyperfine ground-state Zeeman transitions. The ensemble is surrounded by a two-layer mu-metal magnetic shield. The $\mathrm{CPT}$ resonance is monitored by detecting the laser power transmitted through the cell using a low-noise photodiode. The laser is frequency-stabilized near the center of the homogeneously broadened absorption line by modulating the DFB laser current at $60 \mathrm{kHz}$ and demodulating it with the lock-in amplifier LA1. The atomic clock closedloop operation is achieved by a conventional synchronous modulation-demodulation technique $(\sim 1 \mathrm{kHz}$ frequency) applied to the local oscillator frequency through the lockin amplifier LA2.

\section{ExPERImental Investigations And Results}

A typical CPT resonance observed in the Cs-Ne cell for $T_{\text {cell }}=80^{\circ} \mathrm{C}$ is reported in the inset of Fig. 1. The resonance lineshape is well fitted by a Lorentzian function. The full-width at half-maximum (FWHM) is measured to be $1.6 \mathrm{kHz}$ in correct agreement with theoretical values. From [16], the hyperfine coherence relaxation rate $\gamma_{2}$ is calculated to be $4391 \mathrm{rad} / \mathrm{s}$ and the optical resonance homogeneous broadening $\Gamma^{*} / 2 \pi$ is $862 \mathrm{MHz}$. At $80^{\circ} \mathrm{C}$, contributions to the CPT linewidth from wall-collisions and spin-exchange collisions are about equal and clearly dominant over buffer gas collisions. The contrast is measured to be about $1.25 \%$.

Fig. 2 shows both the CPT resonance contrast and the clock frequency shift versus the cell temperature. The $\mathrm{CPT}$ contrast is maximized in the $74^{\circ} \mathrm{C}$ to $81^{\circ} \mathrm{C}$ temperature range. A bell-shaped curve, already observed in [16], [25], [26], is obtained. It is explained by an increase of the alkali atomic density for temperatures up to $80^{\circ} \mathrm{C}$. For higher temperatures, the vapor medium becomes optically thick and causes a strong reduction of the CPT signal. In [16], with a vertical-cavity surface-emitting laser (VCSEL) emitting at $852 \mathrm{~nm}$, the CPT signal maximum with tem- 
perature was measured to be in the $55^{\circ} \mathrm{C}$ to $60^{\circ} \mathrm{C}$ range for quite similar Cs-Ne microcells (pressure and dimensions). This can be qualitatively explained by intrinsic differences between $\mathrm{D}_{1}$ and $\mathrm{D}_{2}$ excitation lines hyperfine structures. The Cs $\mathrm{D}_{2}$ line excited state is composed of 4 hyperfine levels with a frequency separation $(\sim 150$ to $250 \mathrm{MHz})$ much lower than buffer-gas-induced optical broadening in microfabricated cells. Both hyperfine levels of the Cs $\mathrm{D}_{1}$ line are well-resolved and separated by about $1.16 \mathrm{GHz}$. Also, some excited states on the $\mathrm{Cs} \mathrm{D}_{2}$ line couple to only one of the ground states and act as loss-channels for the CPT interaction. Consequently, optical absorption is stronger for the $\mathrm{Cs}_{2}$ line and the alkali vapor becomes optically thick for lower temperatures than in the $\mathrm{D}_{1}$ line case. Moreover, we confirmed that the Cs clock frequency temperature-dependence is canceled close to $80^{\circ} \mathrm{C}$ in the presence of Ne. Using coefficients published in [8], a fit of experimental points gives a Ne pressure of 79 Torr. This cell allows the overlap of a zero-sensitivity of the clock frequency to temperature variations and a maximum of CPT contrast close to $80^{\circ} \mathrm{C}$. This will lead to mutual improvements of the clock long-term and short-term frequency stability.

Combined with a greatly reduced sensitivity of the clock frequency to temperature variations, the light shift is continuously minimized using a method inspired by [21]. For this purpose, the laser power is slowly modulated at $15 \mathrm{~Hz}$ (12\% depth) using a voltage-controlled liquid crystal display (LCD) attenuator. This laser power modulation is converted across the cell through the light shift effect into a slight clock frequency modulation that can be easily detected at the error signal output of the lockin amplifier LA2 used to stabilize the LO frequency. This modulation signal is found to vanish close to a so-called RF power zero-light-point driving the EOM. An additional servo loop based on the lock-in amplifier LA3 is even-

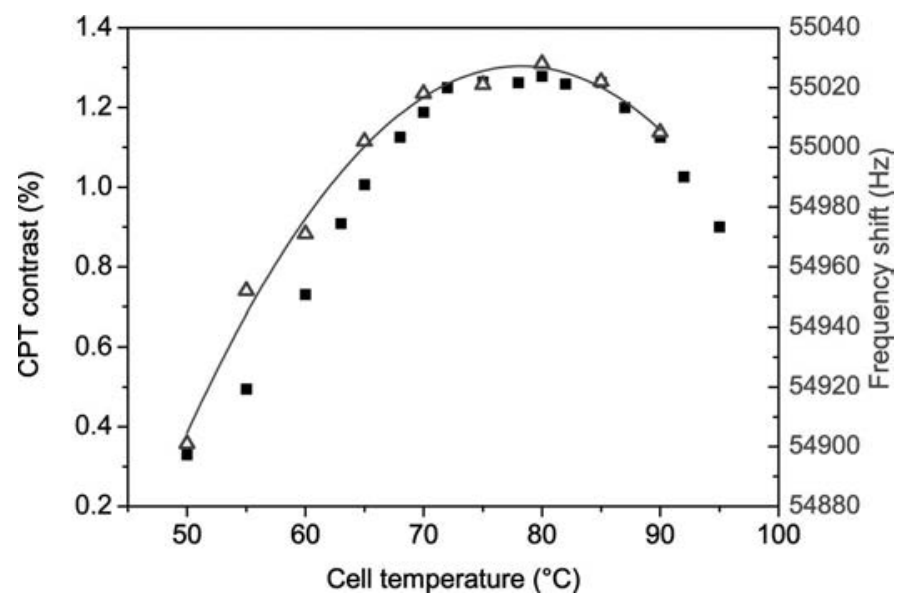

Fig. 2. Coherent population trapping (CPT) resonance contrast (filled squares) and clock frequency shift (triangles) versus the cell temperature. The solid line is a second-order polynomial function fit to the frequency data. For a given temperature, each frequency shift is found from an extrapolation to zero intensity of the light shift curve. The CPT contrast is found to be optimized close to the clock frequency inversion temperature.

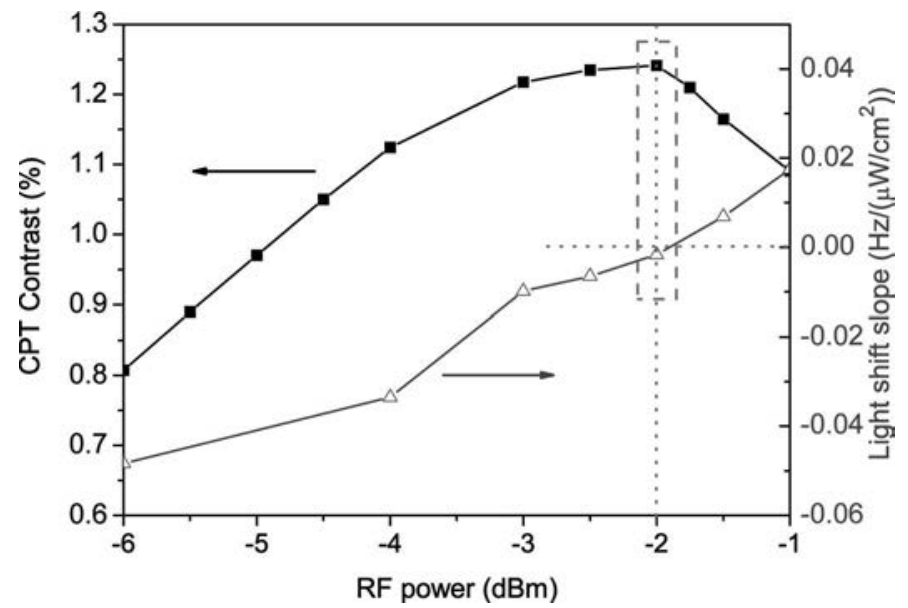

Fig. 3. Coherent population trapping (CPT) resonance contrast (filled squares) and light shift slope (triangles) versus the RF modulation power. The indicated RF power is before amplification of $24 \mathrm{~dB}$. Close to $P_{\mathrm{RF}}=-2 \mathrm{dBm}$, the $\mathrm{CPT}$ resonance contrast is maximized and the light shift can be cancelled. The cell temperature is $80^{\circ} \mathrm{C}$. For each RF power, the frequency shift is measured for 5 to 6 different laser intensity values and is found to be well approximated by a linear function. Each triangle point corresponds to the slope of this function.

tually added to lock the RF power point where no light shift is present. For illustration, Fig. 3 plots both the light shift slope and the CPT resonance contrast versus the RF modulation power.

The light shift slope is measured to be negative for $P_{\mathrm{RF}}$ $<-2 \mathrm{dBm}$, positive for $P_{\mathrm{RF}}>-2 \mathrm{dBm}$ and can be nulled by finely adjusting the $\mathrm{RF}$ power to $P_{\mathrm{RF}} \sim-2 \mathrm{dBm}$. Moreover, as shown in Fig. 3, this RF power coincides with the one maximizing the $\mathrm{CPT}$ resonance contrast. It corresponds well to the RF power maximizing the optical power contained in the two resonant light fields. Such behavior was also reported in [21].

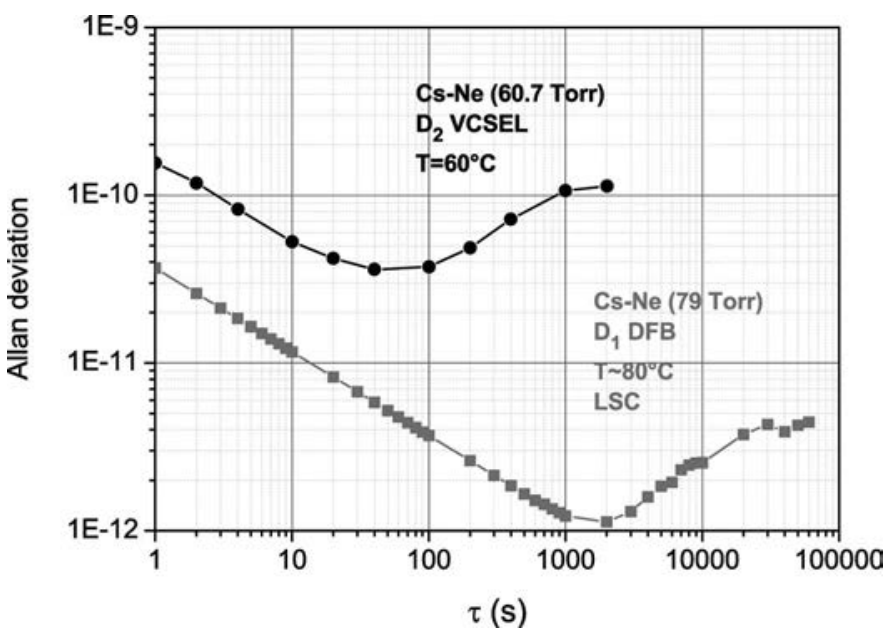

Fig. 4. Allan deviation of the Cs coherent population trapping (CPT) clock based on a Cs-Ne microcell. (circles): vertical-cavity surface-emitting laser (VCSEL) $\mathrm{D}_{2}$ line, no light shift correction, $T_{\text {cell }}=60^{\circ} \mathrm{C}, \mathrm{Ne}$ pressure $=60.7$ Torr $[16]$; (squares): distributed feedback (DFB) $\mathrm{D}_{1}$ line, light shift correction (LSC), $T_{\text {cell }} \simeq 80^{\circ} \mathrm{C}$, Ne pressure $=79$ Torr. 
Fig. 4 reports the frequency stability (Allan deviation) of the CPT clock based on a Cs-Ne microcell. It is measured by comparison with a reference hydrogen maser. Recent results obtained with a $\mathrm{D}_{2}$-line VCSEL and a CsNe microcell $(P=60.7$ Torr $)$ are reported for comparison [16]. In the present case, the short-term frequency stability is measured to be $3.8 \times 10^{-11}$ at $1 \mathrm{~s}$. It is in agreement with a measured atomic Q-factor of $5.7 \times 10^{6}$ and a clock resonance signal-to-noise ratio of 4500 in a $1 \mathrm{~Hz}$ bandwidth. We measured that our laser power modulation for the light shift correction degrades the optimal short-term stability by a factor of about 1.5. The Allan deviation curve decreases well with a $\tau^{-1 / 2}$ slope until $1000 \mathrm{~s}$. After $1000 \mathrm{~s}$, the Allan deviation increases again with a $\tau^{1 / 2}$ slope corresponding to a random-walk frequency noise. The frequency stability is found to be $4 \times 10^{-12}$ at 40000 $\mathrm{s}$. These results obtained with optimal laboratory-scale electronics are comparable to those presented in [3], [22].

\section{CONClusions}

We developed a compact prototype CPT clock based on a Cs-Ne microfabricated vapor cell with an externally modulated DFB diode laser. Advanced techniques such as the use of the $\mathrm{D}_{1}$ line laser resonance, low-noise microwave local oscillator, clock frequency temperature-dependence cancellation and light-shift reduction techniques were implemented. The clock frequency stability is close to $1 \times$ $10^{-12}$ at $2000 \mathrm{~s}$ and $4 \times 10^{-12}$ at $50000 \mathrm{~s}$. Despite the fact that these results do not report a fully-integrated clock, they prove for the first time the potential of the Cs- $\mathrm{Ne}$ microcell solution to develop miniature atomic clocks with high long-term frequency stability and confirm that our original Cs microcell technology is compatible with such developments.

\section{ACKNOWLEDGMENTS}

The authors greatly thank E. De Clercq (LNE-SYRTE, Paris, France) for fruitful discussions and careful reading of the article.

\section{REFERENCES}

[1] J. Vanier, "Atomic clocks based on coherent population trapping: A review," Appl. Phys. B, vol. 81, no. 4, pp. 421-442, 2005.

[2] S. Knappe, V. Shah, P. D. D. Schwindt, L. Hollberg, J. Kitching, L. A. Liew, and J. Moreland, "A microfabricated atomic clock," Appl. Phys. Lett., vol. 85, no. 9, pp. 1460-1462, 2004.

[3] R. Lutwak, A. Rashed, M. Varghese, G. Tepolt, J. LeBlanc, M. Mescher, D. K. Serkland, K. M. Geib, G. M. Peake and S. Römisch, "The chip-scale atomic clock: Prototype evaluation," in Proc. 39th Annu. Precise Time and Time Interval Meeting, 2007, pp. 269-290.

[4] Symmetricom Inc. (2012, Sep.) SA.45s CSAC Chip-scale atomic clock. [Online]. Available: http://www.symmetricom.com/ products/quantum-atomic-oscillators/chip-scale-atomic-clock-csac/ SA.45s-CSAC/ (see datasheet of SA.45s CSAC product)

[5] R. H. Dicke, "The effect of collisions upon the Doppler width of spectral lines," Phys. Rev. Lett., vol. 18, no. 2, pp. 472-475, 1953.

[6] M. Arditi and T. R. Carver, "Pressure, light and temperature shifts in optical detection of $0-0$ hyperfine resonance of alkali metals," Phys. Rev., vol. 124, no. 3, pp. 800-809, 1961.
[7] J. Vanier, R. Kunski, N. Cyr, J. Y. Savard, and M. Tetu, "On hyperfine frequency shifts caused by buffer gases: Application to the optically pumped passive rubidium frequency standard," J. Appl. Phys., vol. 53, no. 8, pp. 5387-5391, 1982.

[8] O. Kozlova, S. Guérandel, and E. De Clercq, "Temperature and pressure shifts of the Cs clock transition in the presence of buffer gases: Ne, $\mathrm{N}_{2}$, Ar," Phys. Rev. A, vol. 83, no. 6, art. no. 062714, 2011.

[9] J. Kitching, S. Knappe, and L. Hollberg, "Miniature vapor-cell atomic-frequency references," Appl. Phys. Lett., vol. 81, no. 3, pp. 553-555, 2002.

[10] L. A. Liew, S. Knappe, J. Moreland, H. Robinson, L. Hollberg, and J. Kitching, "Microfabricated alkali atom vapor cells," Appl. Phys. Lett., vol. 84, no. 14, pp. 2694-2696, 2004.

[11] S. Knappe, V. Gerginov, P. D. D. Schwindt, V. Shah, H. Robinson, L. Hollberg, and J. Kitching, "Atomic vapor cells for chip-scale atomic clocks with improved long-term frequency stability," Opt. Lett., vol. 30, no. 18, pp. 2351-2353, 2005.

[12] A. Douahi, L. Nieradko, J. C. Beugnot, J. Dziuban, H. Maillotte, S. Guérandel, M. Moraja, C. Gorecki, and V. Giordano, "Vapour microcell for chip scale atomic frequency standard," Elec. Lett., vol. 43 , no. 5 , pp. $279-280,2007$.

[13] M. Hasegawa, R. K. Chutani, C. Gorecki, R. Boudot, P. Dziuban, V. Giordano, S. Clatot, and L. Mauri, "Microfabrication of cesium vapor cells with buffer gas for MEMS atomic clocks," Sens. Actuators A, vol. 167, no. 2, pp. 594-601, 2011.

[14] D. Miletic, P. Dziuban, R. Boudot, M. Hasegawa, R. K. Chutani, G. Mileti, V. Giordano, and C. Gorecki, "Quadratic dependence on temperature of Cs $0-0$ hyperfine resonance frequency in single $\mathrm{Ne}$ buffer gas microfabricated vapor cell," Electron. Lett., vol. 46, no. 15, pp. 1069-1071, 2010.

[15] O. Kozlova, R. Boudot, S. Guérandel, and E. De Clercq, "Temperature dependence cancellation of the Cs clock frequency in the presence of Ne buffer gas," IEEE Trans. Instrum. Meas., vol. 60, no. 7, pp. 2262-2266, 2011.

[16] R. Boudot, P. Dziuban, M. Hasegawa, R. K. Chutani, S. Galliou, V. Giordano, and C. Gorecki, "Coherent population trapping resonances in Cs-Ne vapor microcells for miniature clocks applications," J. Appl. Phys., vol. 109, no. 1, art. no. 014912, 2011.

[17] M. Zhu and L. S. Cutler, "Theoretical and experimental study of light-shift in a CPT-based Rb vapor cell frequency standard," in Proc. 32nd Annu. Precise Time and Time Interval (PTTI) Meeting, 2000, pp. 311-323.

[18] F. Levi, A. Godone, and J. Vanier, "The light-shift effect in the coherent population trapping cesium maser," IEEE Trans. Ultrason. Ferroelectr. Freq. Control, vol. 47, no. 2, pp. 466-470, 2000.

[19] V. Gerginov, S. Knappe, V. Shah, P. D. D. Schwindt, L. Hollberg, and J. Kitching, "Long-term frequency instability of atomic frequency references based on coherent population trapping and microfabricated vapor cells," J. Opt. Soc. Am. B, vol. 23, no. 4, pp. 593-597, 2006.

[20] E. E. Mikhailov, T. Horrom, N. Belcher, and I. Novikova, "Performance of a prototype atomic clock based on lin||lin coherent population trapping resonances in Rb atomic vapor," J. Opt. Soc. Am. B, vol. 27, no. 3, pp. 417-422, 2010.

[21] V. Shah, V. Gerginov, P. D. D. Schwindt, S. Knappe, L. Hollberg, and J. Kitching, "Continuous light-shift correction in modulated CPT clocks," Appl. Phys. Lett., vol. 89, art. no. 151124, Oct. 2006.

[22] S. Knappe, V. Shah, V. Gerginov, A. Brannon, L. Hollberg, and J. Kitching, "Long-term stability of NIST chip-scale atomic clock physics packages," in Proc. 38th Annu. Precise Time and Time Interval (PTTI) Meeting, 2008, pp. 241-250.

[23] B. H. McGuyer, Y. Y. Jau, and W. Happer, "Simple method of light shift suppression in optical pumping systems," Appl. Phys. Lett., vol. 94 , no. 25 , art. no. $251110,2009$.

[24] R. Boudot, S. Guérandel, and E. De Clercq, "Simple design low noise NLTL-based frequency synthesizers for a CPT Cs clock," IEEE Trans. Instrum. Meas., vol. 58, no. 10, pp. 3659-3665, 2009.

[25] S. Knappe, J. Kitching, L. Hollberg, and R. Wynands, "Temperature dependence of coherent population trapping resonances," Appl. Phys. B, vol. 74, no. 3, pp. 217-222, 2002.

[26] N. Castagna, R. Boudot, S. Guérandel, E. De Clercq, N. Dimarcq, and A. Clairon, "Investigations on continuous and pulsed interrogation for a CPT atomic clock," IEEE Trans. Ultrason. Ferroelectr. Freq. Contr., vol. 56, no. 2, pp. 246-253, 2009. 